García Medina, M.A., Acosta Meza, D., Atencia Andrade, A. \& Rodríguez Sandoval, M. (2020). Identificación del pensamiento crítico en estudiantes universitarios de segundo semestre de la Corporación Universitaria del Caribe (CECAR). Revista Electrónica Interuniversitaria de Formación del Profesorado, 23(3), 133-147.

DOI: https://doi.org/10.6018/reifop.435831

\title{
Identificación del pensamiento crítico en estudiantes universitarios de segundo semestre de la Corporación Universitaria del Caribe (CECAR)
}

María Angélica García Medina, David Acosta Meza, Asdrúbal Atencia Andrade, Marco Rodríguez Sandoval

Corporación Universitaria del Caribe (Colombia)

\section{Resumen}

La investigación tiene como objetivo analizar el pensamiento crítico en estudiantes universitarios de segundo semestre de la Corporación Universitaria del Caribe CECAR. Se realizó una aproximación teórica sobre el constructo pensamiento crítico y como este es valorado por diversos autores. La investigación es de corte cuantitativo-descriptivo, con una muestra intencional de 100 estudiantes pertenecientes a los programas de Ciencias del Deporte, Ingenierías y Licenciatura en Lingüística y Literatura. Se utiliza el test Evaluación del Pensamiento Crítico Contextualizado (EP-2C) propuesto por Carrasco (2018) que consta de cinco variables y 26 reactivos diseñados para medir las variables de estudio: análisis, interpretación, evaluación, inferencia y explicación. Los resultados arrojan que los estudiantes están en el nivel de PC mediamente logrado en el cual son capaces de argumentar acercándose levemente a la respuesta esperada. Esto indica que se deben diseñar estrategias en ambientes enriquecidos y secuencias didácticas que propendan por el fortalecimiento de las variables estudiadas.

\section{Palabras clave}

Pensamiento crítico; estrategias; habilidades; estudiantes.

\section{Contacto:}

María Angélica García, maria.garciame@cecar.edu.co, carretera Troncal de Occidente Km. 1, Vía Corozal - Sincelejo, Colombia.

Artículo resultado de los avances del proyecto de investigación: Modelo didáctico para el fortalecimiento del pensamiento crítico, con tecnologías emergentes, de los estudiantes universitarios del Caribe colombiano. 


\title{
Identification of critical thinking in second semester university students of the Caribbean University Corporation (CECAR)
}

\begin{abstract}
The research aims to analyze critical thinking in second semester university students of the Caribbean University Corporation (CECAR). A theoretical approach was made to the critical thinking construct and how various authors value it. The research is quantitative and descriptive, with an intentional sample of 100 students belonging to the programs of Sports Sciences, Engineering and Linguistics and Literature. The test Evaluation of Critical Contextualized Thinking (EP-2C) proposed by Carrasco (2018) is used. It consists of five variables and 26 reagents designed to measure the study variables: analysis, interpretation, evaluation, inference and explanation. The results show that the students are at the medium level of CP in which they are able to argue by getting slightly closer to the expected response. This indicates that strategies should be designed in enriched environments and didactic sequences that tend to strengthen the variables studied.
\end{abstract}

\section{Key words}

Critical thinking; strategies; skills; students.

\section{Introducción}

En la actualidad, las instituciones de educación superior están enfrentando un grave problema que es la falta de pensamiento crítico en los estudiantes, el cual es fundamental para que ellos se conviertan en profesionales reflexivos y críticos que puedan desenvolverse competentemente en el contexto laboral.

Para Herrero (2016), pensar críticamente es "saber pensar, decidir en qué creer o qué hacer, es una técnica común a todos los estudiantes, independientemente de su especialidad" ( $p$. 13); mientras que para Saiz (2019), "pensar críticamente es alcanzar la mejor explicación para un hecho, fenómeno o problema con en el fin de saber resolverlo eficazmente" (p.19). Por consiguiente, este autor afirma que a los alumnos se les debe orientar para que potencien sus capacidades intelectuales, con el fin de que expliquen los fenómenos del contexto, emitan juicios, argumenten y conceptualicen. Es por esto que Sainz, asume su estudio desde los dominios específicos del conocimiento y parte de cuatro categorías: argumentación, metacognición, emociones y solución de problemas.

Por su parte, Ocampo y Tamayo (2018) señalan que la formación de pensamiento crítico en la escuela requiere de esfuerzos conjuntos, intencionados y prolongados en el tiempo, para lo cual es imprescindible el uso de estrategias de enseñanza - aprendizaje que les ayude a los educandos a desarrollar plenamente las habilidades básicas de pensamiento para luego dirigirse hacia las habilidades de pensamiento superior.

De igual forma, Saiz y Rivas (2017) comentan que se requieren sistemas evaluativos eficaces y válidos que permitan examinar de la manera más precisa las habilidades de PC y se ajusten a los objetivos de las intervenciones. Es por esto que ellos consideran que primero se debe efectuar un diagnóstico en el que se identifiquen las carencias y los obstáculos que dificultan el proceso de aprendizaje, luego hacer una intervención con estrategias dinámicas y, por último, evaluar el impacto de la aplicación de estas en los discentes y el alcance del PC. 
Teniendo en cuenta lo anterior, el objetivo de este estudio se centró en analizar los niveles de pensamiento crítico (PC) de los estudiantes del II semestre de CECAR que pertenecen a los programas de Ciencias del Deporte, Ingenierías y Licenciatura en Lingüística y Literatura. Para alcanzar este propósito, en primer lugar, se realizó una revisión conceptual sobre el pensamiento crítico, sus niveles y formas de medición, en segundo lugar, se determinaron los niveles de PC que presentan los grupos de estudiantes mencionados anteriormente, y por último se llevó a cabo una valoración de resultados en el marco de las variables evaluadas y los referentes teóricos pertinentes. Cabe anotar que los resultados del diagnóstico serán el insumo para continuar con la segunda fase del estudio, en la que se diseñarán las estrategias para fomentar las habilidades de PC de acuerdo con el contexto y las necesidades de los educandos.

\section{Conceptualización sobre el pensamiento crítico}

Revisando los diferentes conceptos de PC en los diversos contextos investigativo, se hallaron semejanzas y divergencias entre los teóricos que los definen. Dewey (1989), se refiere al pensamiento crítico como "pensamiento reflexivo, el cual supone un estado de duda, de vacilación, de perplejidad, de dificultad mental, en el cual se origina el pensamiento, y un acto de busca, de caza, de investigación para encontrar algún material que esclarezca la duda, que disipe la perplejidad" (como se citó en Marciales, 2003).

Para Facione (2007), el pensamiento crítico "es el juicio auto regulado y con propósito que da como resultado interpretación, análisis, evaluación e inferencia, como también la explicación de las consideraciones de evidencia, conceptuales, metodológicas, criteriológicas o contextuales en las cuales se basa ese juicio" (p. 21). En correspondencia con lo anterior, Guzmán y Sánchez (2006) describen el PC como:

Un proceso intencionado de pensamiento que está orientado al logro de una meta, como el tipo de pensamiento que se utiliza en la resolución de problemas, la toma de decisiones, el análisis y las inferencias lógicas. De alguna manera, todas estas definiciones implican procesos mentales que son útiles para una tarea cognitiva particular" (p. 5)

Valenzuela, Nieto y Saiz (2011) manifiestan que las personas piensan críticamente cuando intentan resolver un problema, evaluar un argumento, decidir sobre una creencia o tomar una decisión en general. Para lograr estos fines, "se evalúan no solo los productos y resultados del pensamiento, es decir, creencias, elecciones, conclusiones, hipótesis, etc., sino también los procesos que los han generado, es decir, el razonamiento que condujo a tales conclusiones" (p. 826).

Desde la perspectiva del desarrollo biológico de los seres humanos, López, Martínez y Sierra (2017), sitúan el pensamiento crítico en la última etapa de desarrollo propuesta por Piaget, la cual se caracteriza por la presencia de operaciones intelectuales formales que facilitan la inserción afectiva e intelectual de los individuos en el mundo adulto, a partir del uso de la lógica y el establecimiento de conclusiones abstractas que presuponen un recurso metacognitivo.

Desde la perspectiva del conocimiento, se busca que el PC se fomente a través de estrategias dinámicas que favorezcan las habilidades de pensamiento. Es así como Halpern (2014) describe el PC como el uso de habilidades o estrategias cognitivas que aumentan la probabilidad de un resultado deseable:

Se utiliza para describir el pensamiento que tiene un propósito, un razonamiento y una meta dirigida: el tipo de pensamiento involucrado en la resolución de problemas, 
la formulación de inferencias, el cálculo de probabilidades y la toma de decisiones, cuando el pensador está usando habilidades que son reflexivas y efectivas para el contexto particular y tipo de tarea de pensamiento (p. 8).

\section{Valoración de los niveles de pensamiento crítico}

En diversos estudios realizados en el contexto universitario colombiano en los que se han valorado los niveles de pensamiento crítico de los discentes, se ha concluido que estos presentan un desempeño bajo. Verbigracia, Mota (2010) expresa que los universitarios poseen muchos vacíos en cuanto al desarrollo del pensamiento crítico y esto se refleja en su habilidad de comprensión lectora, pues no pueden hacer un análisis eficiente de los textos escritos. Igualmente, él anota que los datos arrojados en su investigación refuerzan los resultados obtenidos por los estudiantes universitarios en las pruebas nacionales Saber-Pro $(2015,2018)$, puesto que sus niveles de pensamiento crítico fueron bajos, tal como lo informa el Ministerio de Educación Nacional de Colombia.

Laiton (2010) comenta que lo deseable es encontrar en las aulas de clases, en los primeros semestres universitarios, estudiantes críticos, pensantes, analíticos que estén ávidos de conocimiento, que pregunten e indaguen sobre él y que valoren el aprendizaje en sus proyectos de vida; pero que esto no se evidencia en el contexto colombiano.

Domínguez (1999, como se citó en Zabala, 2013) señala que uno de los objetivos de las instituciones de educación superior debe ser generar talentos para el mercado laboral. No obstante, él plantea que esto no es fácil, ya que los discentes presentan serias dificultades no solo para desarrollar las competencias genéricas, las cuales son requeridas hoy para la formación integral; sino también los niveles de pensamiento crítico que son indispensables para que los individuos sean capaces de comparar, contrastar, tomar decisiones, juzgar y evaluar.

\section{Metodología}

\section{Diseño}

La investigación es de corte descriptivo, puesto que se determinó el nivel de las habilidades de pensamiento crítico en los estudiantes de segundo semestre, casi en los inicios de su carrera profesional; y de carácter cuantitativo, debido a que lo anterior se llevó a cabo haciendo una medición de variables e indicadores con base en una escala numérica a través de una prueba estandarizada.

\section{Participantes}

La investigación se realizó en una institución educativa colombiana de carácter privado llamada: Corporación Universitaria del Caribe (CECAR). La muestra fue intencional e igual a la población, pues se escogieron los 100 estudiantes de segundo semestre que hacen parte de los programas de Ciencias del Deporte (27), Ingenierías (33) y Licenciatura en Lingüística y Literatura (40). Se seleccionaron estos sujetos porque se supone que ya han tenido un proceso de adaptación al contexto universitario.

\section{Instrumento}

Para determinar los niveles de PC en los estudiantes de segundo semestre, se utilizó el Test EP-2C de Carrasco (2018). Para su elaboración, este autor llevó a cabo una revisión de la literatura especializada con respecto al pensamiento crítico y el entono educativo; y a partir de allí tomó las ideas de Facione (2007) en cuanto a las variables que subyacen al pensamiento crítico; las de Paul y Elder (2005) referentes a los niveles de desarrollo del 
pensamiento crítico; y las de Kohlberg (1984) respecto al test de desarrollo moral, específicamente, lo relacionado con la categorización de respuestas particulares.

El test está constituido por 26 reactivos, los cuales fueron construidos para medir las variables de estudio: análisis, interpretación, evaluación, inferencia y explicación. Además, fue hecho con base en reactivos de preguntas abiertas. Para la revisión de cada una de las preguntas, se elaboró una categorización de desempeño a nivel argumentativo. Este tipo de categorización de respuesta está basado en la lógica de que no existen respuestas acertadas ni erróneas, sino más bien la capacidad de argumentar una postura ante la interrogante propuesta (Carrasco, 2018).

El EP-2C plantea un esquema de puntuación de respuestas en el que se establece una categorización de desempeño argumentativo de los sujetos con base en una postura fundamentada en relación con lo que pregunta (Carrasco, 2018). El puntaje se basa en los siguientes tres niveles:

- Logrado (2 puntos): cuando el sujeto es capaz de argumentar de manera coherente y clara, acercándose a la respuesta esperada satisfactoriamente.

- Medianamente Logrado (1 punto): cuando el sujeto es capaz de argumentar acercándose levemente a la respuesta esperada.

- No Logrado (o puntos): cuando el sujeto no es capaz de argumentar de manera coherente y clara u omite la respuesta.

\section{Procedimiento}

El test EP-2C, se aplicó a los estudiantes de segundo semestre, después de haberles explicado el objetivo del mismo, su organización y la forma como debían realizarlo.

Para el analizar los datos arrojados por el Test, se usó el software estadístico Epi Info 7.2.3.1. Por medio de este se correlacionaron tres variables (género, edad y tipo de escuela) con el fin de caracterizar la población objeto de estudio. Asimismo, se realizó la correspondencia entre las variables del test: análisis, interpretación, evaluación, inferencia y explicación.

\section{Resultados}

\section{Caracterización de la población objeto de estudio}

Con respecto al género de los sujetos de estudio, en la figura 1, se evidencia que el $47 \%$ de ,os sujetos evaluados corresponde al género femenino y el $53 \%$ al género masculino.

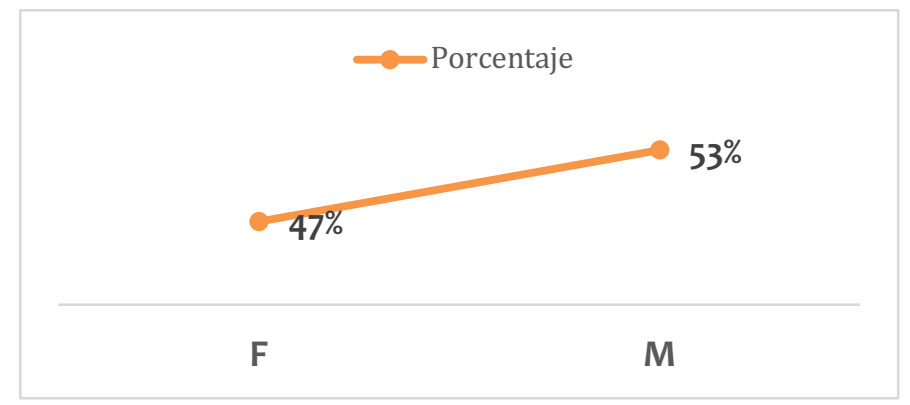

Figura 1. Género de los sujetos investigados.

En lo referente a las edades de los sujetos de estudio, en la figura 2, se aprecia que esta es heterogénea, ya que se ubican en un rango entre los 17 y los 32 años. 


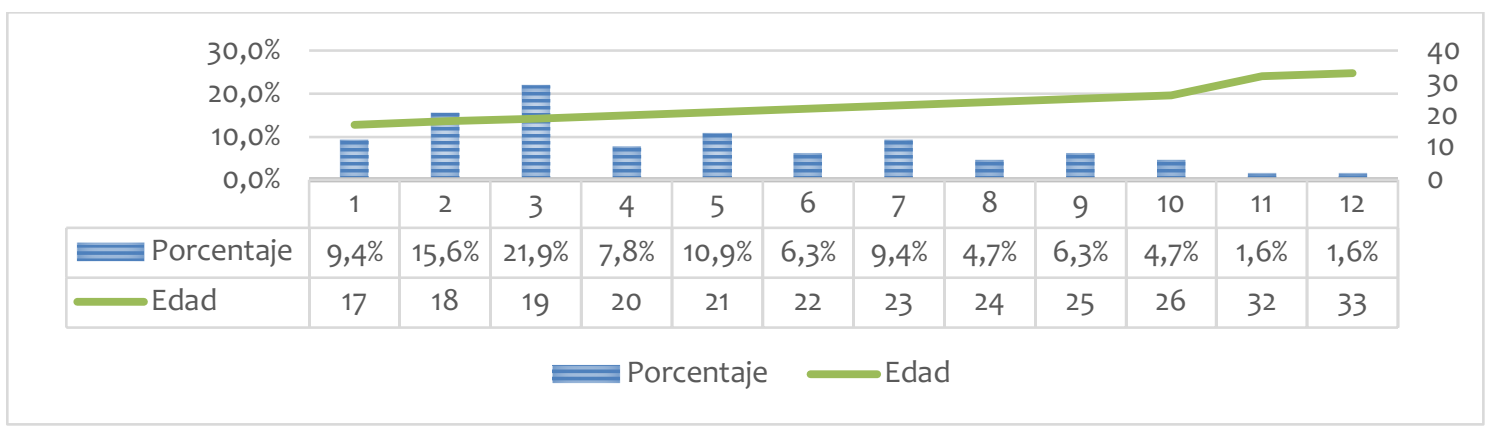

Figura 2. Edades de los sujetos investigados. Elaboración propia.

Con respecto a la relación entre edad y género, en la figura 3, se observa que el rango de edad preponderante tanto en las mujeres como en los hombres oscila entre los 20 y 25 años.
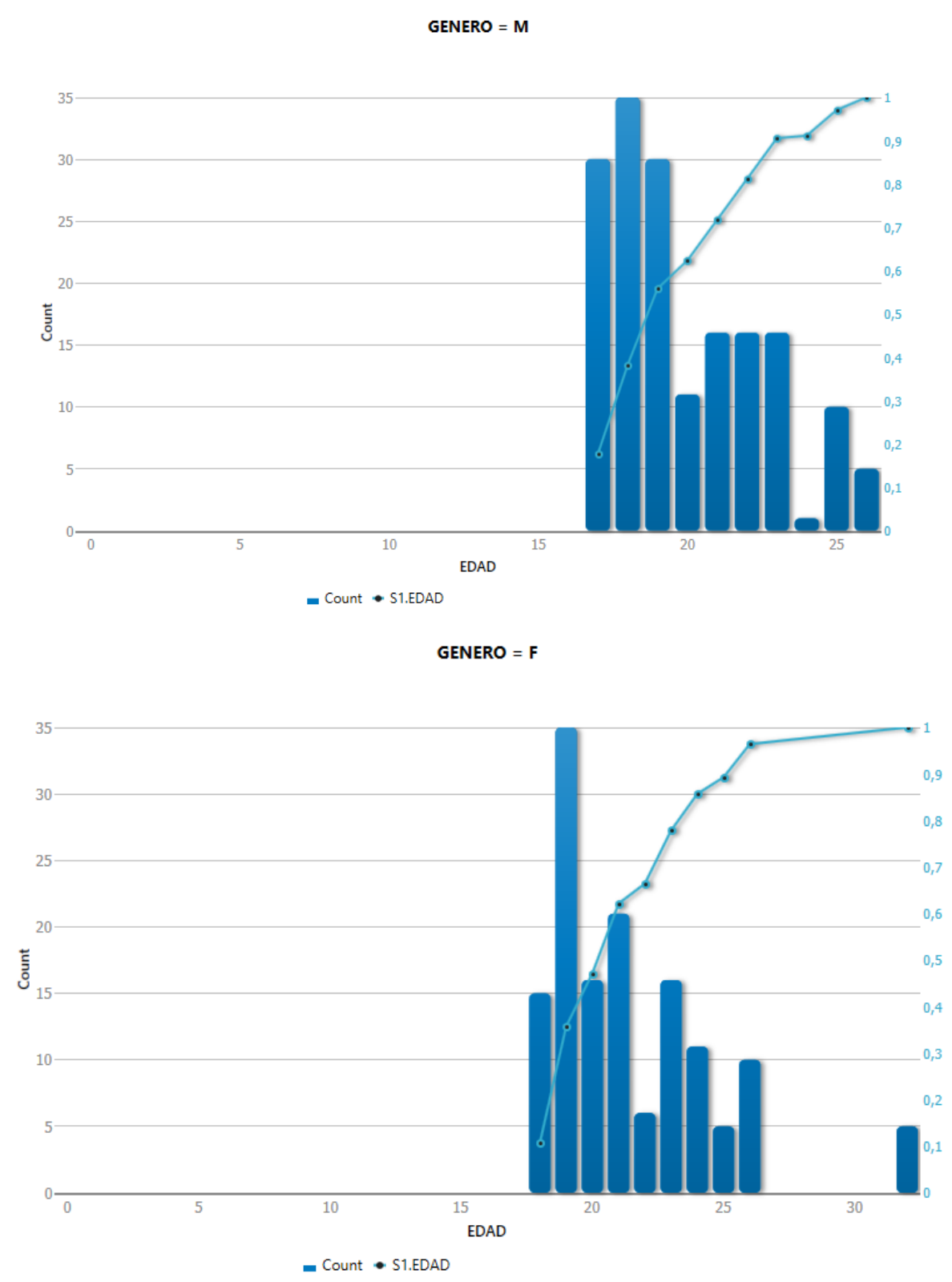

Figura 3. Relación entre género y edad. 
En la figura 4, se aprecia que el $82,81 \%$ de los estudiantes finalizó los estudios de la básica secundaria en una escuela pública; mientras que el 17,19\%, en una escuela privada. Los datos anteriores podrían ser luego estudiados para establecer si el tipo de educación que se recibe en una escuela pública o privada influye en la apropiación de las habilidades de análisis, interpretación, evaluación, inferencia y explicación del pensamiento crítico.

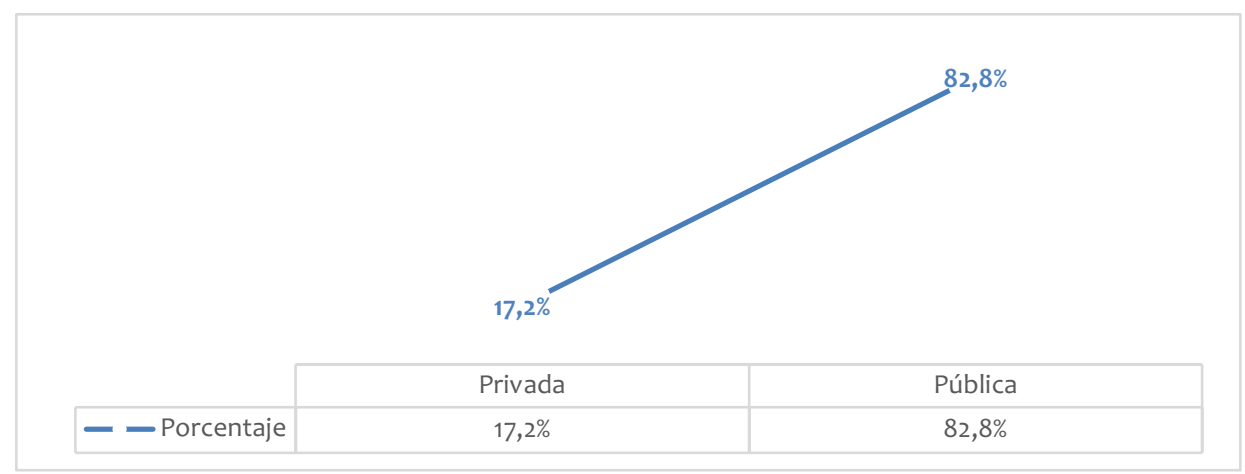

Figura 4. Tipo de escuela a la que asistió el participante. Elaboración propia.

\section{Evaluación del pensamiento crítico}

\section{Valoración de la variable análisis}

En la figura 5, se observa que el $74,60 \%$ de los estudiantes obtuvieron un no logrado en la variable análisis, lo que indica que no poseen un nivel de análisis para dar respuesta al planteamiento del contexto del ítem establecido.

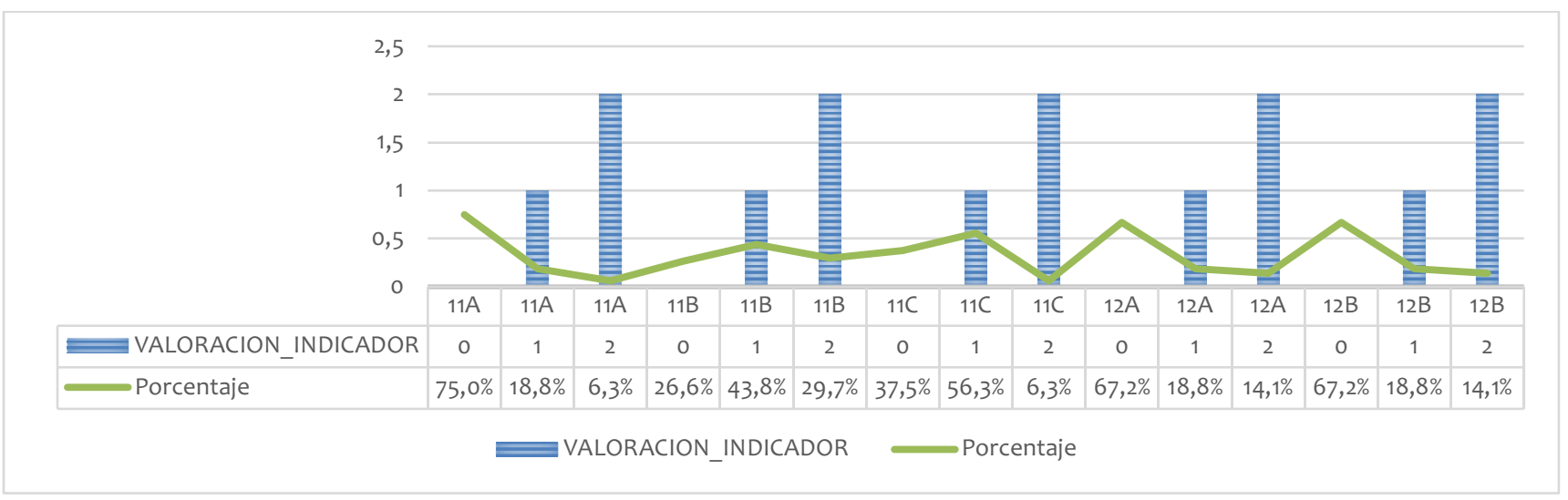

Figura 5. Porcentaje de repuesta variable análisis. o: no logrado 1: Medianamente Logrado 2: Logrado.

La variable análisis obtuvo un $77 \%$ de individuos ubicados en el rango no logrado, un $20 \%$ en el rango medianamente logrado y tan solo el 3\% en el rango logrado. 


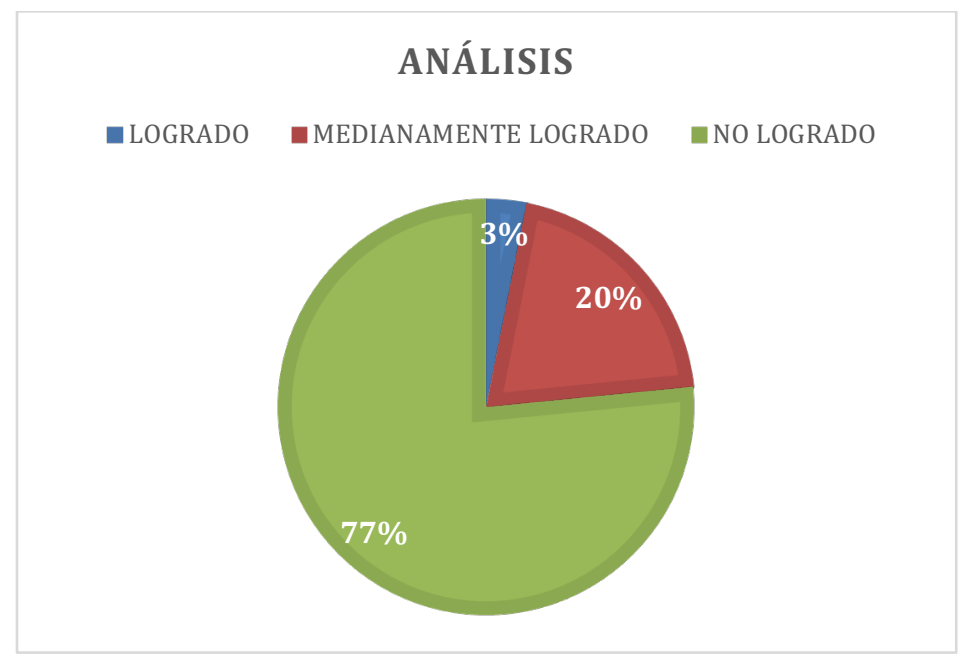

Figura 6. Resultado general variable análisis. Elaboración a partir de los datos.

\section{Valoración de la variable interpretación}

En la figura 7, se muestra que el desempeño de los estudiantes en el indicador de resolución de las preguntas se aproximó al nivel 1: mediamente logrado. De los anterior se deriva que los estudiantes se encuentran en un nivel medio para interpretar textos partiendo de un contexto dado.

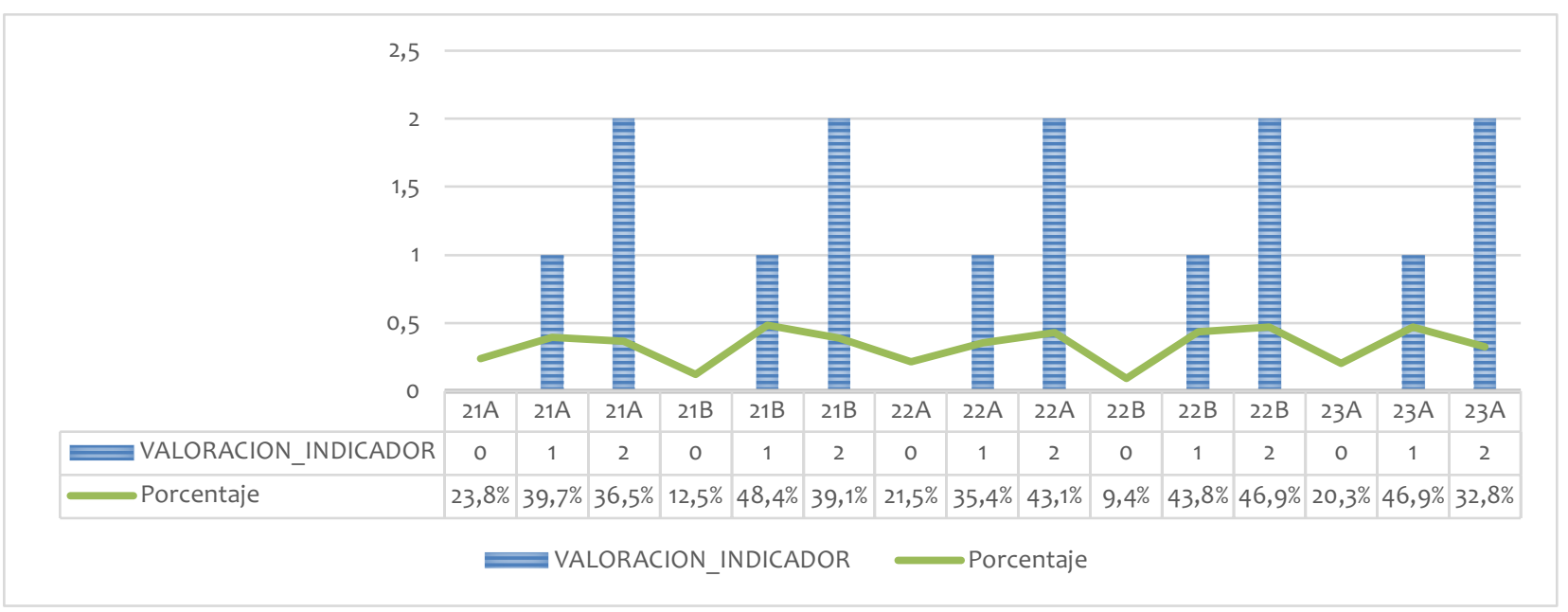

Figura 7. Porcentaje de repuesta variable interpretación. 0: no logrado 1: medianamente logrado 2: logrado

Con respecto a la variable interpretación, en la figura 8 , se muestra que el $50 \%$ de la población objeto de estudio se ubica en el rango medianamente logrado, el $34 \%$ en el rango logrado y tan solo el $16 \%$ en el rango no logrado. 


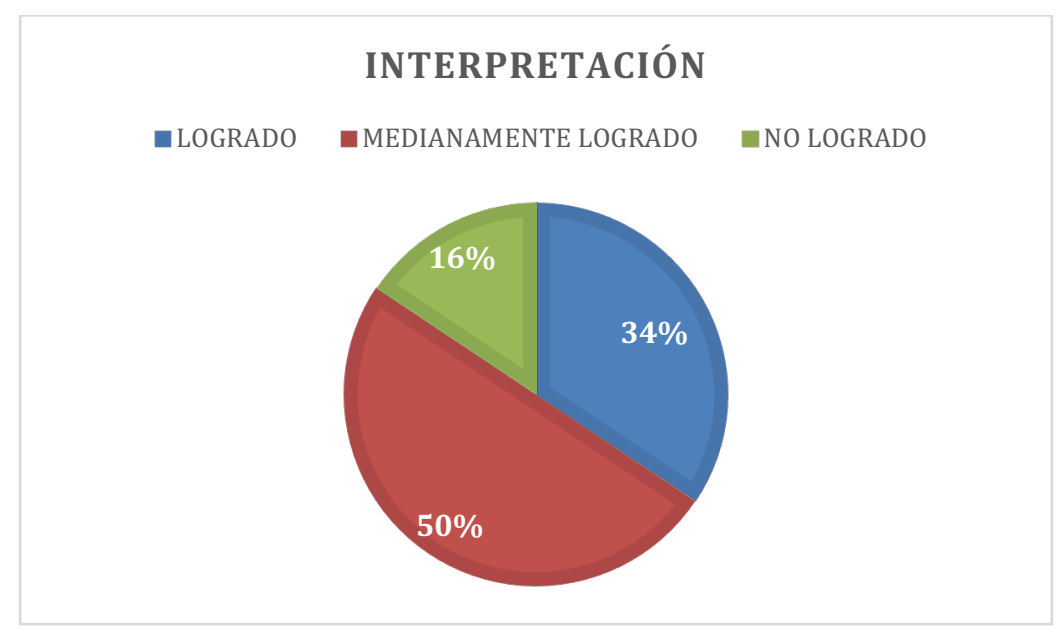

Figura 8. Resultado general de la variable interpretación. Elaboración a partir de los datos

\section{Valoración de la variable evaluación}

En la figura 9, se observa que el desempeño de los estudiantes en la resolución de las preguntas alcanzó el nivel 1: mediamente logrado. Se infiere, entonces, que los estudiantes se encuentran en un nivel medio para evaluar textos.

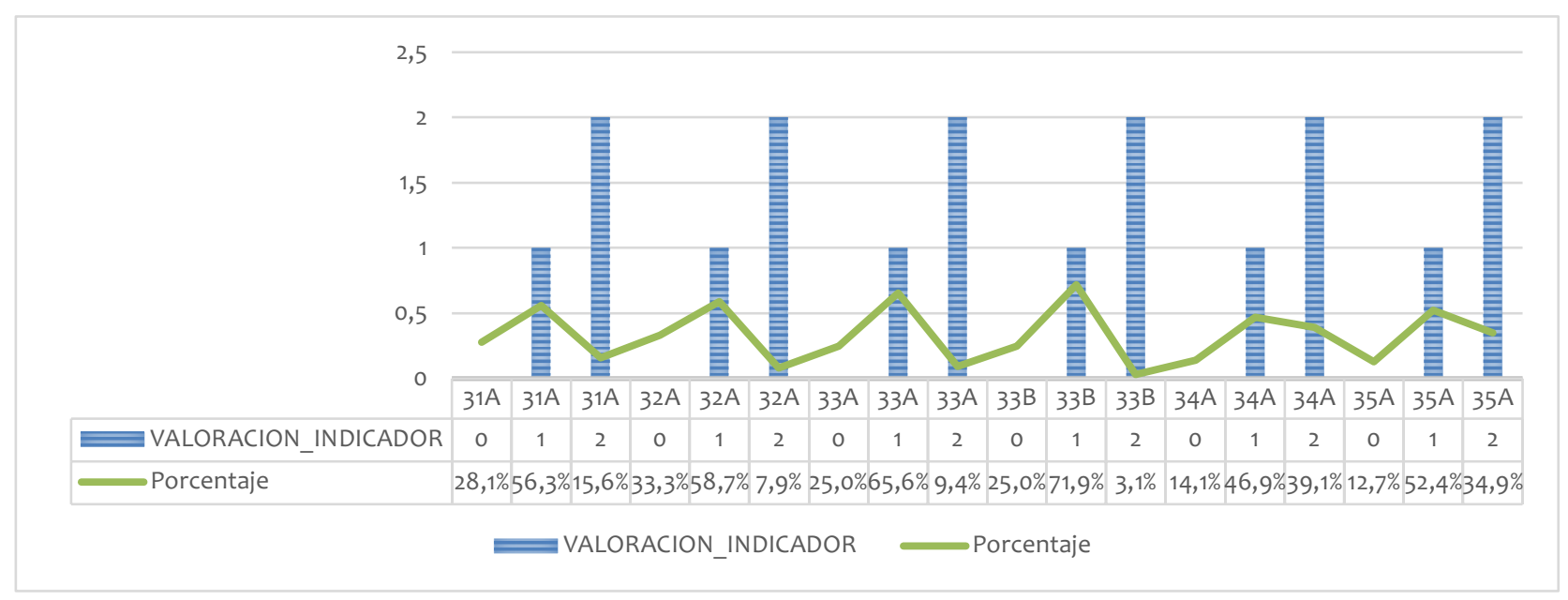

Figura 9. Porcentaje de repuesta variable evaluación. o: no logrado 1: Medianamente Logrado 2: Logrado

A nivel general, para la variable evaluación, se observa que de la población objeto de estudio el $65 \%$ de ésta se ubica en el rango medianamente logrado, el $19 \%$ en el rango logrado y el $16 \%$ en el rango no logrado. 


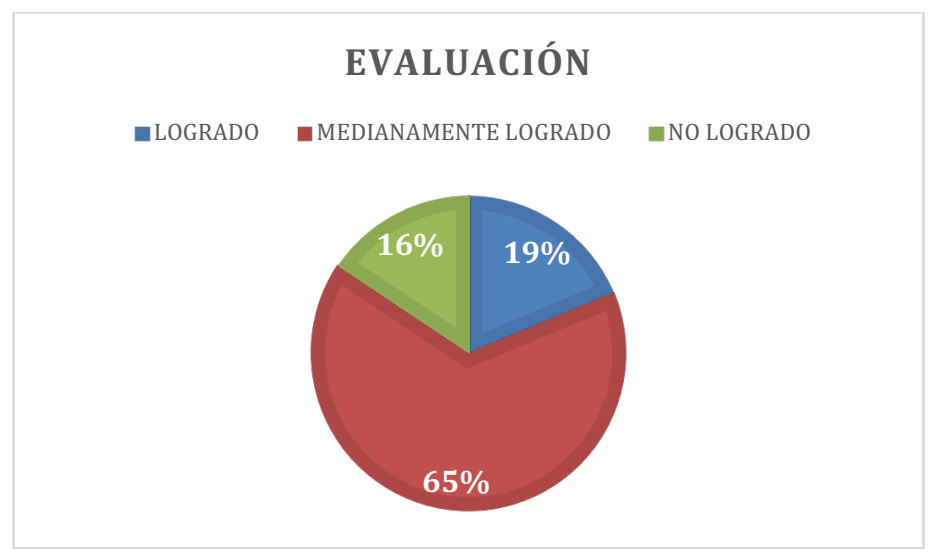

Figura 10. Resultados generales de la variable evaluación. Elaboración a partir de los datos

\section{Valoración de la variable Inferencia}

En la figura 11, se aprecia que el desempeño de los estudiantes en la resolución de las preguntas alcanzó el nivel 1: mediamente logrado. Es decir, que los estudiantes presentan un nivel medio al hacer inferencias a partir del análisis de la información.

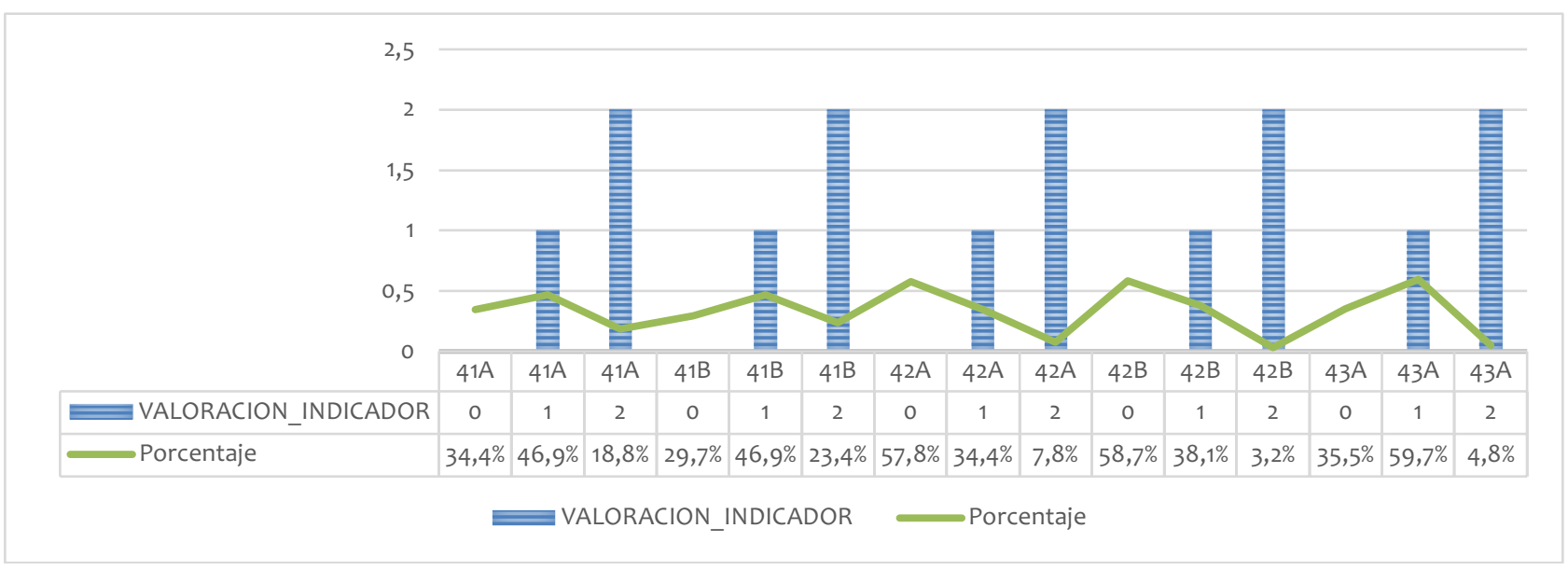

Figura 11. Porcentaje de repuesta variable inferencia. o: no logrado 1: Medianamente Logrado 2: Logrado

En cuanto a la variable inferencia, el $61 \%$ de la población se situó en el nivel no logrado y el $39 \%$, en el mediamente logrado.

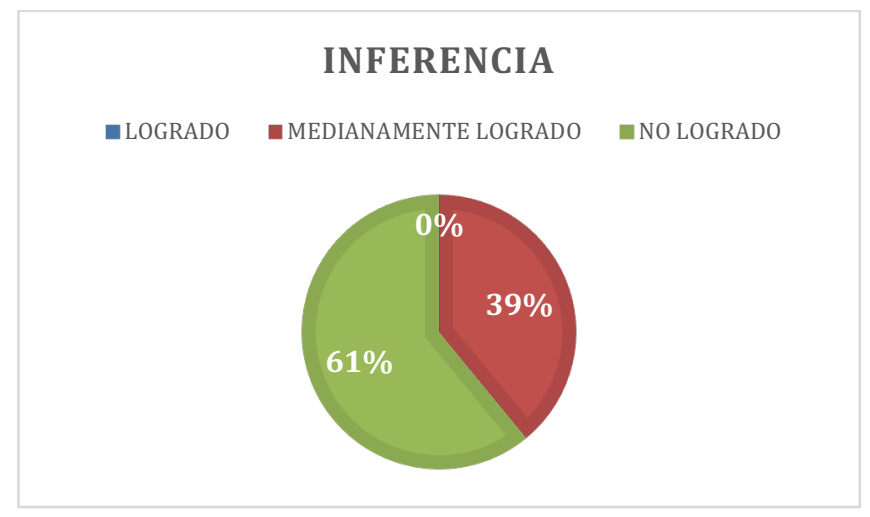

Figura 12. Resultados generales de la variable inferencia. Elaboración a partir de los datos 


\section{Valoración de la variable explicación}

En la figura 13, se muestra que el desempeño de los estudiantes en la resolución de las preguntas se situó en el nivel 1: mediamente logrado. De lo anterior se desprende, que los estudiantes alcanzan un nivel medio para explicar textos.

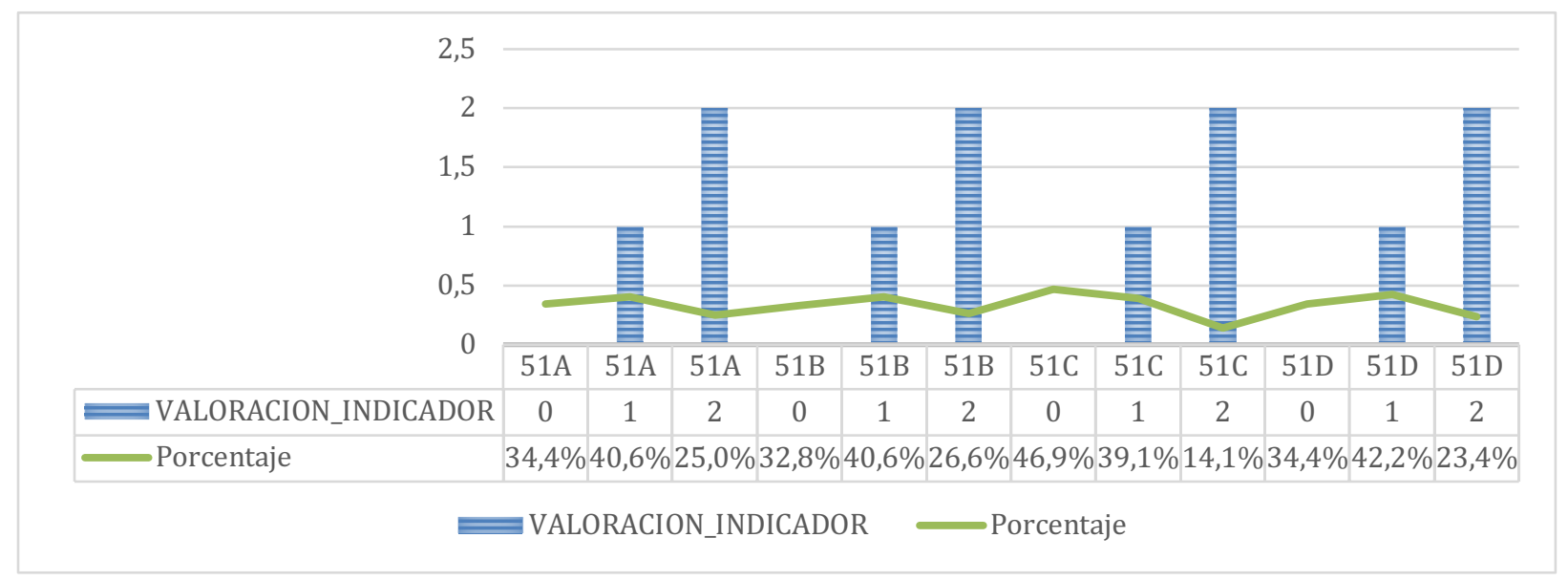

Figura 13. Porcentaje de repuesta variable explicación. o: no logrado 1: Medianamente Logrado 2: Logrado

Con respecto a la variable explicación, en la figura 14 , se observa que el $67 \%$ de la población objeto de estudio se ubica en el rango no logrado, el $34 \%$ en el medianamente logrado y tan solo el $2 \%$ se ubicó en el rango logrado.

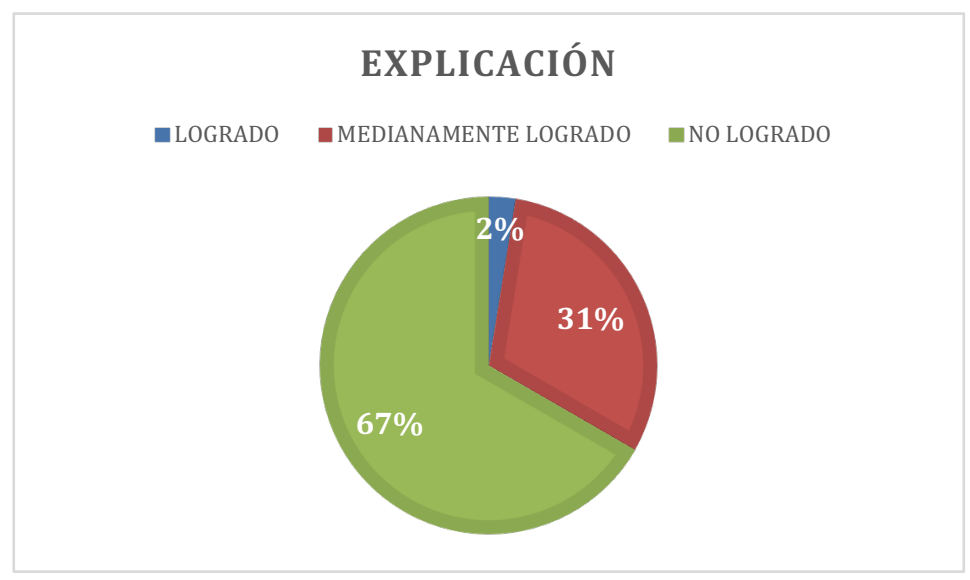

Figura 14. Resultados generales de la variable explicación. Elaboración a partir de los datos.

En términos generales, se resalta que el $64 \%$ de la población objeto de investigación se ubica en el nivel medianamente logrado de PC, el $34 \%$ en el no logrado y tan solo el $2 \%$ en el logrado. El Nivel del PC de la población estudiantil es mediamente logrado en todas las variables: análisis, interpretación, evaluación, inferencia y explicación (ver la figura 15). 


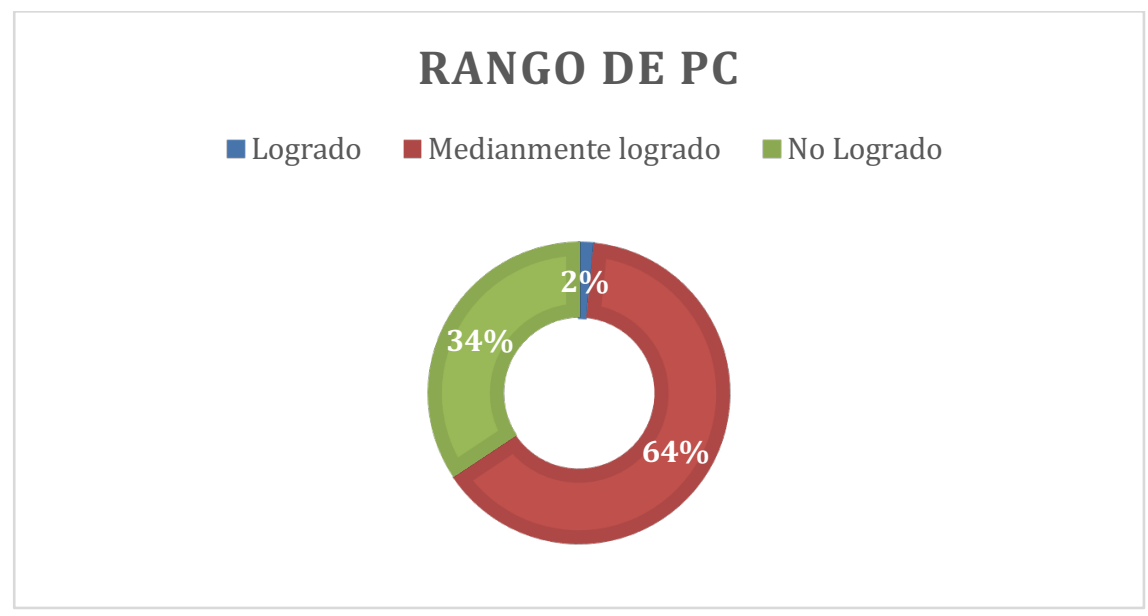

Figura 15. Rango de PC de la población objeto de estudio.

\section{Discusión y conclusiones}

Los procesos básicos de pensamiento se entienden como la capacidad de procesar información y construir conocimientos adaptados a necesidades y limitaciones. En ese sentido, Meyer (1983, como se citó en la Universidad Internacional de la Rioja, 2019) indica que suceden cambios en una persona cuando es capaz de resolver un problema, debido a que las habilidades se activan de forma automática y sin que el individuo sea consciente. Además, él expresa que el pensamiento se determina como natural si una persona establece las razones por las cuales toma determinadas decisiones ante situaciones particulares en los niveles de análisis, reflexión, argumentación y justificación. Esto es lo que lleva al individuo a desarrollar un pensamiento crítico, tal como lo establece López (2003) quien identifica el PC como un proceso por el cual una persona reflexiona sobre diferentes aspectos o experiencias de la vida cotidiana para resolver un problema, emitir una postura y orientar las respuestas de los propios comentarios.

Los resultados demuestran que la población objeto de estudio posee representaciones mentales que de alguna manera afectan de forma positiva o negativa el alcance del pensamiento crítico. En la investigación se evidencia que un porcentaje alto de estudiantes terminan sus estudios secundarios en escuelas públicas ubicadas en regiones de difícil acceso o en condiciones no favorables para el desarrollo de fundamentos conceptuales básicos, lo cual se refleja en las bajas puntuaciones en las habilidades de análisis, interpretación, evaluación, inferencia y explicación. De forma similar, Castellón (2018) define el PC como un proceso por el cual una persona piensa reflexivamente sobre diferentes aspectos, situaciones o experiencias de su vida cotidiana para resolver un problema y adoptar una postura crítica y reflexiva en diferentes contextos sociales. Igualmente, él establece que el docente en el salón de clase juega un papel importante al momento de promover preguntas reflexivas.

Al determinar el rango de edad, se encontró un contraste entre las edades: la mínima edad es de 17 años y la máxima de 32 años, con un rango medio de 20 a 25 años. Los estudiantes en este rango medio son capaces de establecer ideas lógicas y abstractas. Piaget (1997) plantea que una de las fases del desarrollo humano es la en la etapa de operaciones formales que abarca desde los 12 años hasta la vida adulta, en la cual se organiza un sistema complejo de lógica y de ideas abstractas. Al relacionar este principio teórico con los resultados de la 
investigación, se encuentra que los estudiantes de CECAR obtuvieron mejores puntuaciones en las preguntas relacionadas con sentido lógico en contraste con las de la variable explicación, puesto que las premisas establecidas no connotaban argumentos que sustentaran o evidenciaran el carácter explicativo de éstas.

La variable análisis es la capacidad de identificar las relaciones de inferencia reales y supuestas entre enunciados, preguntas, conceptos, descripciones u otras formas de representación que tienen el propósito de expresar creencias, juicios, experiencias, razones, información u opiniones (Facione, 2007, como se citó en Carrasco, 2018). Acorde con lo anterior, en los resultados de la investigación se encontró que el $77 \%$ de los estudiantes no logró el desempeño argumentativo, el $20 \%$ lo logró medianamente y el $3 \%$ lo logró completamente. Con esto se concluye que los estudiantes se alejan de la capacidad de análisis, dado que los argumentos expuestos por los estudiantes en las respuestas no muestran una relación entre las bases de las hipótesis establecidas. Entonces, para que un estudiante alcance la habilidad de análisis debe "identificar los elementos del objeto a analizar; determinar los criterios de descomposición del todo; relacionar dichos elementos y reproducir las relaciones" (Velázquez, Remolina y Calle, 2013, p. 29).

La variable interpretación se concibe como comprender y expresar el significado o la relevancia de una amplia variedad de experiencias, situaciones, datos, eventos, juicios, convenciones, creencias, reglas, procedimientos o criterios (Facione, 1990, como se citó en Carrasco, 2018). Relacionando este concepto con los resultados del desempeño argumentativo, se encuentra que el $50 \%$ de los estudiantes se ubica en el nivel medianamente logrado, el $34 \%$ en el logrado y el $16 \%$ en el no logrado. Esto lleva a determinar que la población estudiantil es capaz de interpretar situaciones basadas en datos, juicios, convicciones, creencias, criterios o procedimientos.

La variable evaluación está definida como la valoración de la credibilidad de los enunciados o de otras representaciones que recuentan o describen la percepción, experiencia, situación, juicio, creencia u opinión de una persona; y la valoración de la fortaleza lógica de las relaciones de inferencia, reales o supuestas, entre enunciados, descripciones, preguntas u otras formas de representación (Facione, 2007, como se citó en Carrasco, 2018). En esta variable el desempeño en la población estudiantil alcanza el nivel medianamente logrado con el $65 \%$, el logrado con el $19 \%$ y el nivel no logrado con el $16 \%$. Se deduce a partir de estos resultados que los estudiantes son capaces de evaluar medianamente las relaciones que se establecen entre argumentos, pero que sus explicaciones o descripciones no poseen fuerza lógica que permita visionar la intencionalidad de la declaración establecida.

La variable inferencia parte de la identificación y aseguramiento de elementos necesarios para sacar conclusiones razonables, formular conjeturas e hipótesis, considerar la información pertinente y sacar las consecuencias que se desprendan de los datos, enunciados, principios, evidencias, juicios, creencias, opiniones, conceptos, descripciones, preguntas u otras formas de representación (Facione, 2007, como se citó en Carrasco, 2018). En esta variable los estudiantes presentaron un desempeño argumentativo de $61 \%$ en el nivel no logrado y de $39 \%$ en el nivel medianamente logrado. Esto ocurre principalmente en los reactivos que incluían la formulación de conjeturas e hipótesis carentes de juicios concretos y descripciones para dar conclusiones pertinentes.

La variable explicación es considerada como la capacidad de presentar los resultados del razonamiento propio de manera reflexiva y coherente (Facione, 2007, como se citó en Carrasco, 2018). En esta variable los estudiantes alcanzaron un desempeño argumentativo de $67 \%$ en el nivel no logrado, de $31 \%$ en el medianamente logrado y de $2 \%$ en el logrado. Esto 
se refleja en los argumentos establecidos en los reactivos planteados que carecen de un razonamiento deductivo y no muestran evidencias de conceptos apropiados.

En conclusión, al determinar el nivel de PC alcanzado por los estudiantes mediante el desempeño argumentativo se encuentra que este fue medianamente logrado por el $64 \%$ de ellos. Por consiguiente, se proponen el diseño de estrategias en ambientes enriquecidos y secuencias didácticas en los que se implementen procedimientos para potenciar habilidades de pensamiento crítico.

\section{Referencias}

Carrasco, J. (2018). Medición del desarrollo del pensamiento crítico en estudiantes chilenos/as de Educación Superior. (Tesis de maestría). Universidad de Concepción, Concepción, Chile.

Castellón, S. (27 de agosto de 2018). El pensamiento crítico. Oriente Informa. (898), p. 11. Recuperado de http://www.cchoriente.unam.mx/gaceta/Gacetas\%202018/Oriente\%20Informa\%20898.pdf.

Facione, P. (2007). Pensamiento crítico: ¿Qué es y por qué es importante? Insight assessment, 22. Recuperado de http://www.eduteka.org/PensamientoCriticoFacione.php

Guzmán, S. y Sánchez, P. (2006). Efectos de un programa de capacitación de profesores en el desarrollo de habilidades de pensamiento crítico en estudiantes universitarios en el Sureste de México. Revista Electrónica de Investigación Educativa, 8 (2). Recuperado de http://redie.uabc.mx/vol8noz/contenido-guzman.html

Halpern, D. (2014). Thought and knowledge: an introduction to critical thinking. New York, USA: Psychology Press.

Laiton, I. (2001). Formación de pensamiento crítico en estudiantes de primeros semestres de educación superior. Revista Iberoamericana de Educación, 53(3), 2-7. https://doi.org/10.35362/rie5331730

López, M. (2003). Pensamiento crítico y creatividad en el aula. Santa Fe de Bogotá: Editorial Trillas.

López, E., Martínez, Y, y Sierra, I. (2017). Pensamiento crítico. Reto formativo para docentes de bachilleres. Revista Espacios, 38(60). Recuperado de http://www.revistaespacios.com/a17v38n60/17386034.html

Marciales, G. (2003). Pensamiento crítico: diferencias en estudiantes universitarios en el tipo de creencias, estrategias e inferencias en la lectura crítica de textos. (Tesis doctoral). Universidad Complutense de Madrid, Madrid, España.

Mota, C. (2010). Desarrollo del pensamiento crítico a través del discurso argumentativo: Una experiencia pedagógica en un curso de lectura y escritura. Entre Lenguas, 15. 11-23. Recuperado de https://dialnet.unirioja.es/servlet/articulo?codigo $=3620700$

Ocampo, E. y Tamayo, Ó. (2018). Representaciones de aprendizaje en estudiantes que participan en un curso sobre teorías del aprendizaje. Revista Latinoamericana de Estudios Educativos, 14 (2), 238-268.

Piaget, J. (1997). El preadolescente y las operaciones proporcionales. Psicología del niño. Madrid, España: Ediciones Morata S.L.

Saiz C. (2019). Pensamiento crítico y eficacia. Madrid: Editorial Pirámide. 
Sainz, C., y Rivas, S. F. (2017). New teaching techniques to improve critical thinking. The DIAPROVE methodology. Educational Research Quarterly, 40(1), 3-36.

Universidad Internacional de la Rioja. (2019). Habilidades de pensamiento. Material no publicado.

Valenzuela, J., Nieto, A. y Sainz, C. (2011). Critical thinking motivational scale: A contribution to the study of relationship between critical thinking and motivation. Electronic Journal of Research in Educational Psychology, 9(2), 823-848. Recuperado de https://eric.ed.gov/?id=EJ946122

Velázquez, B., Remolina, N. y Calle, M. (2013). Habilidades de pensamiento como estrategia de aprendizaje para los estudiantes universitarios. Revista de investigaciones UNAD, 12(2), 23-41. Recuperado de http://hemeroteca.unad.edu.co/index.php/revista-deinvestigaciones-unad/article/view/1174.

Zabala, S. (2013). Perfil por competencias del profesional de la informática: una visión desde la perspectiva del mercado empleador venezolano. Revista Espacios, 34 (6). Recuperado de http://www.revistaespacios.com/a13v34no6/13340607.html 